\title{
A Radiologia no Início do Século XXI
}

Radiology at the Beginning of the 21st Century

Pedro Alves ${ }^{1,2}$

\section{INTRODUÇÃO}

Em novembro de 1895 Willem Conrad Roentgen fez a famosa descoberta de uma nova forma de radiação invisível a que chamou raio-X, cuja propriedade mais marcante era a capacidade de atravessar materiais que não deixavam passar a luz visível.

A radiologia nasceu nesta data, e rapidamente disseminou-se pelo mundo, dado que os aparelhos necessários à produção dos raios-X e usados por Roentgen (tubos de Crookes) existiam em múltiplos laboratórios hospitalares e universitários.

Durante a maior parte do século XX, a radiologia foi uma especialidade dedicada à obtenção de informação anatómica e morfológica dos tecidos e órgãos. Nas últimas duas décadas do século XX e nas primeiras décadas do século XXI, a imagiologia funcional, molecular e radiologia de intervenção vieram alterar o paradigma da especialidade.

A disseminação de sistemas informáticos com maior capacidade computacional permitiu o tratamento e análise
Autor Correspondente:

Pedro Alves [pedro.alves@jmellosaude.pt] R. Mário Botas, 1998-018 Lisboa, Portugal

rotineiros de volumes crescentes de dados e imagens em formato digital bem como uma maior facilidade de partiIha de informação quer a uma escala regional quer global.

A maior resolução espacial das técnicas imagiológicas, combinada com o desenvolvimento de biomarcadores e métodos de aquisição de imagem com informação funcional, aumentaram exponencialmente o volume e o tipo de informações passíveis de serem obtidas pelos modernos exames imagiológicos.

É sempre difícil prever o futuro de uma especialidade médica, mas atualmente podem identificar-se várias tendências e campos de expansão na área da Imagiologia..,2

\section{INTELIGÊNCIA ARTIFICIAL}

Os métodos de inteligência artificial analisam grandes quantidades de dados, identificam padrões e características semelhantes associando uma capacidade de aprendizagem progressiva do computador que faz a análise e que com treino adequado adquire a capacidade de analisar novos conjuntos de dados a partir dos originais. Estes métodos são usados atualmente para reconhecimento automático de imagens, texto e voz. 
Os algoritmos de "deep learning" (DL) têm-se tornado progressivamente mais sofisticados e são especialmente eficazes e superiores ao desempenho humano em tarefas de reconhecimento de padrões, segmentação de imagem e deteção de objetos. $\bigcirc$ desenvolvimento inicial destas técnicas foi no mercado dos videojogos, mas rapidamente foi adaptado à radiologia.

Os algoritmos de DL constituem um conjunto de técnicas, das quais as mais utilizadas em radiologia são as redes neurais (cuja estrutura imita as redes de neurónios cerebrais) e que foram inicialmente desenvolvidas para tarefas de segmentação de imagem e classificação. A eficácia destas "redes" aumenta com o número de vezes que a informação é introduzida e podem aprender de várias maneiras tratando dados com níveis variáveis de organização e estruturação.

O objetivo em radiologia é de dispor de um sistema computacional "inteligente", capaz de analisar grandes quantidades de dados complexos e identificar padrões clinicamente significativos e úteis.

As primeiras aplicações envolvendo imagem médica ocorreram nos campos da retinopatia diabética, dermatologia e microscopia. Estas áreas prestaram-se bem aos desenvolvimentos iniciais porque envolviam o reconhecimento de padrões numa única imagem ou em pequenos grupos de imagens.

Em radiologia, pelo contrário, a análise envolve centenas de imagens por exame que são heterogéneas, altamente variáveis de doente para doente e refletindo anatomias e patologias diferentes.

O desenvolvimento e investigação dos métodos de inteligência artificial em radiologia estão a concentrar-se em quatro grandes áreas: deteção, quantificação, segmentação de imagem e classificação diagnóstica.

Os primeiros modelos computorizados de deteção lesional foram usados em radiologia torácica para deteção de nódulos pulmonares e apresentavam níveis de sensibilidade próximos dos 70\% valores que com os novos algoritmos subiram para cerca de $90 \%$ e são capazes, autonomamente, de incorporar características lesionais no seu processo de aprendizagem.

A classificação diagnóstica de entidades patológicas e lesões é outra área de investigação, citando-se como exemplos a doença pulmonar obstrutiva crónica em radiologia torácica e a determinação automática da idade óssea em radiologia musculoesquelética.

A quantificação constitui outro campo de estudo em rápido desenvolvimento. Os métodos quantitativos são extremamente úteis no seguimento de lesões focais (nódulos), quantificação de edema ou atrofia (doenças cerebrovasculares, neuromusculares) ou quantificação de metabolitos (ferro hepático), apenas para referir algumas aplicações. Os métodos tradicionais de análise imagiológica envolvem geralmente medições de áreas, volumes ou eixos lesionais. Os novos algoritmos permitem a análise de características tissulares e lesionais não detetadas pelo olho humano, tais como medidas de texturas, histogramas de características imagiológicas, e correlação entre imagens radiológicas e não radiológicas (ex. endoscópicas). Estes métodos já foram testados no seguimento de neoplasias esofágicas com correlação entre imagiologia e dados endoscópicos. Espera-se que o desenvolvimento destas tecnologias permita a identificação de padrões patológicos complexos, não evidentes à análise visual humana ou estatística convencional.

Os processos de aquisição de imagem sofreram uma importante transformação especialmente desde a primeira década do séc. XXI. A imagem adquirida não é mais uma "fotografia" e as imagens digitais são, atualmente, um conjunto de "dados" que podem ser transferidos e manipulados. ${ }^{3}$

Os dados base são assim mais importantes que a imagem convencional e podem ser objeto de tratamento e estudo adicional por software adequado, nomeadamente pelos novos algoritmos de inteligência artificial.

A segmentação imagiológica é também outro foco destes novos algoritmos. Inicialmente a segmentação de imagem era exclusivamente manual, um processo muito laborioso e demorado. Os novos métodos automáticos permitem uma segmentação eficaz e mais rápida, libertando tempo aos profissionais para outras tarefas.

A Inteligência Artificial terá também um papel importante nos métodos de pesquisa de bases de dados, de relatórios e de informação arquivada, mudando o paradigma da pesquisa por palavras-chave e usando algoritmos de linguagem natural que são mais eficazes em detetar e organizar informação relevante.

Existem, contudo, problemas importantes na utilização destes novos métodos e nos processos interpretativos por eles gerados, nomeadamente em relação ao uso de grandes bases de dados para análise (levantando questões de privacidade individual e de acesso aos dados) e à responsabilidade médico-legal das interpretações efetuadas (quem é responsável por uma classificação de um achado como normal ou doença? - o algoritmo ou o médico radiologista?). 


\section{MEDICINA DE PRECISÃO}

A prática de uma medicina de precisão, com a combinação de informação morfológica, funcional e molecular permitirá à radiologia dar um contributo cada vez mais individualizado na caracterização da doença.

A possibilidade de ligar o fenótipo imagiológico e a informação genotípica através, por exemplo, de modalidades híbridas como a tomografia por emissão de positrões-ressonância magnética (PET-RM) permitirá a obtenção de informação cada vez mais individualizada, definindo populações e subpopulações restritas de doentes aos quais se poderão aplicar terapêuticas semelhantes.

A oncologia tem estado na linha da frente para a aplicação destes conceitos. ${ }^{3}$ Os estudos de imagem com biomarcadores moleculares revelam, por exemplo, diferenças da estrutura intratumoral da lesão neoplásica primária e entre lesões secundárias, diferenças essas que não são detetáveis por métodos convencionais de biópsia. Dado que a heterogeneidade estrutural quer dos tumores primários, quer das lesões secundárias é uma das principais barreiras ao tratamento oncológico eficaz, espera-se que o desenvolvimento destes métodos permita uma melhor compreensão da sua fisiopatologia.

O uso de biomarcadores e a combinação de técnicas de biologia molecular nos estudos de imagem necessitam, contudo, de uma estreita colaboração entre os cientistas e os clínicos que incorporarão estes novos resultados no diagnóstico e planeamento terapêutico.

A complexidade dos novos dados obtidos e o volume de informação que está disponível atualmente à escala global exigem modelos organizados de suporte à decisão clínica, e recomendações sobre estes assuntos têm sido publicadas nos últimos anos por várias instituições.

A ausência de metodologias estandardizadas das novas técnicas combinadas de imagiologia e medicina molecular tem sido o maior obstáculo à sua implementação e a uma eficaz partilha de informação a nível global. ${ }^{4}$

\section{RADIOLOGIA E ENVELHECIMENTO POPULACIONAL}

O aumento da esperança de vida trouxe consigo vários desafios para os exames imagiológicos envolvendo a definição de novos critérios e padrões de normalidade para os diferentes grupos etários. Os parâmetros fisiológicos e anatómicos normais para um doente de 50 anos não são idênticos aos de um doente de 90 anos. Serão ne- cessários estudos para definição de novos parâmetros de normalidade dos grupos etários mais extremos.

Inovações da abordagem imagiológica e de estratificação de risco por idades e população serão necessárias juntamente com estratégias de rastreio e diagnóstico clínico. A radiologia de intervenção terá um papel preponderante ao permitir a administração de terapêuticas minimamente invasivas em doentes com maior debilidade e com maior número de comorbilidades.

\section{EVOLUÇÃO TECNOLÓGICA}

Durante a maior parte do século XX a técnica fundamental de aquisição de imagem foi baseada nos RX. A disseminação inicial após a sua descoberta foi muito rápida porque os tubos de vácuo essenciais à sua produção estavam disponíveis em laboratórios de todo o mundo e foi relativamente fácil reproduzir as condições da experiência inicial de Roentgen.

Atualmente, os processos de inovação e desenvolvimento tecnológico em radiologia estão maioritariamente concentrados na indústria privada e menos nos centros académicos. A consequência desta situação é a tendência dominante para desenvolver as técnicas já existentes em detrimento de novas técnicas com outros princípios de aquisição de imagem.

A introdução de novos métodos de imagem é, assim, mais demorada e a pesquisa menos incentivada, dado que o retorno económico é menos seguro.

A inovação em radiologia envolve o desenvolvimento de novos dispositivos de aquisição de imagem (hardware) e o desenvolvimento de software de aquisição e análise de imagem, esta última área com a investigação mais repartida entre centros académicos de investigação e entidades privadas.

Dada a posição de charneira que ocupa entre tecnologia, investigação básica, laboratorial e clínica, a investigação imagiológica beneficia grandemente de uma colaboração inter e multidisciplinar entre médicos e não médicos (físicos, matemáticos, engenheiros, informáticos).

Um problema atual advém da evolução tecnológica muito rápida, cujas novidades são largamente publicitadas pelas marcas fabricantes, antes de haver tempo para uma avaliação do seu benefício clínico real. Várias questões se colocam aos decisores clínicos e administrações hospitalares: valerá a pena comprar tecnologia a um ritmo quase anual se o benefício para os cuidados prestados for marginal? Qual a estratégia mais eficaz para a compra e manutenção de equipamentos visando a atualização constante ao mesmo tempo que não se disper- 
sam recursos em material cujo retorno clínico não é relevante? Como avaliar o real valor clínico e institucional de uma atualização de equipamento sem possibilidade de renovação e acompanhamento tecnológico versus a compra de um equipamento novo com maior potencial de renovação e atualização nos próximos anos? Como garantir que a estratégia de compra de equipamento não está desfasada do clínico da instituição (as novidades das marcas fabricantes são frequentemente aliciantes, mas poderão ser irrelevantes para os objetivos do projeto clínico)? Como implementar as novas técnicas de imagiologia molecular e biomarcadores cujo potencial é enorme, mas que atualmente implicam recursos logísticos e económicos que estão fora do alcance da maioria dos serviços e departamentos hospitalares? Numa era de desenvolvimento acelerado de novas tecnologias e de orçamentos cada vez mais controlados é imperativo que todos os procedimentos efetuados e implementados sejam submetidos a um rigoroso processo de avaliação custo-benefício. Os exames e procedimentos radiológicos devem demonstrar, além do valor diagnóstico "clássico", um impacto real nos tratamentos efetuados, nos resultados clínicos individuais dos doentes e também nos efeitos para a comunidade e sociedade em geral. Questões relacionadas com a dose de radiação cumulativa geral numa população e o impacto de técnicas imagiológicas de rastreio são apenas dois exemplos.

\section{RADIOLOGIA GERAL VERSUS SUBESPECIALIZAÇÃO}

As subespecialidades em radiologia têm já várias décadas, sendo essenciais para uma resposta clínica adequada e um trabalho multidisciplinar de qualidade. ${ }^{5}$ A unidade da disciplina de radiologia é, contudo, também essencial, e os argumentos para um treino básico em radiologia geral antes da subespecialização são vários.

Como exemplo apresenta-se a avaliação de estudos de corpo inteiro que necessita de treino em radiologia geral (usados em oncologia ou patologia sistémica articular, metabólica ou infeciosa com atingimento multifocal).

A prática de uma radiologia de emergência de qualidade implica também um treino adequado em radiologia geral.

Outro exemplo de subespecialização é a radiologia de intervenção que está em crescimento constante com a possibilidade de efetuar terapêuticas minimamente invasivas com o auxílio de meios imagiológicos cada vez mais precisos e com maior resolução espacial. $\bigcirc$ aparecimento de serviços clínicos de radiologia com consultas autónomas, internamento, salas de intervenção e áreas de recobro seria impensável há 30 anos em que se equacionava a especialidade de radiologia com uma atividade exclusivamente diagnóstica e sem contacto clínico.

\section{SEGURANÇA DO DOENTE}

A radioproteção vem à cabeça das questões relacionadas com a segurança do doente num departamento de radiologia. As reduções de dose nos exames foram um dos principais avanços das últimas duas décadas e a Indústria teve um papel fundamental. Doses muito baixas são agora possíveis de maneira rotineira em estudos de tomografia computorizada (TC) (ex.: TC tórax 1 mSV e radiografia do tórax-0,1 mSV).

Estes avanços criaram uma pressão adicional sobre os departamentos de radiologia para uma atualização e renovação de equipamento antiquado incapaz de efetuar estudos de baixas doses bem como uma obrigatoriedade de serem realizados registos cada vez mais precisos das doses de radiação administradas.

Com uma sociedade cada vez mais informada haverá menor tolerância para locais em que os mínimos de dose de radiação não sejam cumpridos ou que seja utilizado equipamento desatualizado e que não acompanha o estado da arte. A obrigatoriedade de publicitar e divulgar as doses usadas nos exames radiológicos conhecerá uma disseminação rápida nos próximos anos e os próprios doentes exigirão que essa informação seja disponibilizada.

\section{INTEGRAÇÃO DO PROCESSO CLÍNICO E IMAGIOLOGIA}

As tecnologias de informação permitem atualmente grandes avanços na organização da informação clínica de um serviço médico, possibilitando a integração de todos os dados do processo clínico.

A integração crescente dos dados clínicos e dos exames complementares permite o desenvolvimento de sistemas mais sofisticados de suporte à decisão clínica bem como uma avaliação mais célere da evidência disponível na literatura.

Esta integração crescente permite ao médico radiologista planear de maneira mais eficaz a estratégia imagiológica de estudo para um doente individual, definindo quais os exames a efetuar e respetiva prioridade.

Os relatórios e o seu formato beneficiam também do desenvolvimento dos sistemas de informação com aparecimento de relatórios estruturados, incorporando imagens-chave, esquemas e formatos multimédia. ${ }^{6}$ 


\section{SUSTENTABILIDADE ECONÓMICA EM RADIOLOGIA}

Tradicionalmente os modelos económicos associados à radiologia têm sido baseados no volume de exames efetuados, não tendo em consideração a qualidade dos procedimentos efetuados quer para o doente, individualmente, quer para a comunidade em que estão integrados.

As técnicas mais complexas são, de um modo geral, mais caras do que os exames mais simples, escondendo atrás da sua complexidade uma frequente irrelevância clínica que estudos mais simples teriam resolvido. Um exemplo clássico em radiologia musculoesquelética é o estudo de lesões focais ósseas, que frequentemente não necessitam mais do que uma série adequada de radiogramas para um diagnóstico correto.

O controle de custos e manutenção da qualidade num cenário de desenvolvimento tecnológico vertiginoso só será possível com modelos económicos que incorporem os resultados clínicos nas estratégias de gestão de equipamentos e de técnicas disponíveis pelos departamentos. Estes aspetos são ainda mais importantes no domínio da medicina de precisão e imagiologia molecular em que os custos de desenvolvimento e implementação de biomarcadores são muito elevados.

Independentemente destes obstáculos, a crescente expetativa dos doentes e da sociedade para uma medicina cada vez mais precisa e eficaz não diminuirá. O foco na qualidade terá de ser prioritário nos processos de organização dos serviços.

Desde o início deste século que a definição de qualidade em radiologia tem sido progressivamente alargada a critérios de valor diagnóstico e terapêutico com uma tendência para uma alteração dos modelos de pagamento e reembolso que contemplarão, cada vez mais, apenas as técnicas e procedimentos que demonstrem um real valor e benefício clínico. Os modelos tradicionais de pagamento baseados no volume, além de sofrerem, desde sempre, de problemas importantes (quantidade sem atenção ao valor/qualidade), estão a ser progressivamente substituídos pela força do desenvolvimento tecnológico mais acelerado e pelo maior controle orçamental quer a nível de entidades públicas quer privadas.

\section{CONCLUSÃO}

A radiologia está a sofrer transformações profundas desde o final do século XX e nos próximos anos prevê-se o aparecimento de múltiplas tecnologias que serão certamente disruptivas, mas que criarão também novas oportunidades.
As tecnologias, protocolos e algoritmos de formação das imagens sofrerão transformações dramáticas, mas a interpretação da informação obtida e correlação com o quadro clínico do doente individual e sua patologia não mudará.

Apesar de todos os avanços tecnológicos as melhores instituições mundiais mantêm os mesmos princípios de atendimento e serviço à pessoa doente. Uma abordagem personalizada por uma equipa de profissionais altamente treinada são as bases de cuidados de saúde eficazes independentemente do contexto institucional em que se trabalha.

Estes princípios são facilmente transportáveis para a área imagiológica e implicam a presença nos departamentos de equipas treinadas para assegurar a adequada realização dos procedimentos e o uso de tecnologia atualizada com protocolos de aquisição de imagem e de intervenção terapêutica otimizados e validados num contexto em que a segurança e a qualidade são os valores fundamentais do serviço a prestar ao doente.

CONFLITOS DE INTERESSE: Os autores declaram não ter qualquer conflito de interesse na realização do presente trabalho.

FONTES DE FINANCIAMENTO: Não houve qualquer fonte de financiamento na realização do presente trabalho.

CONFLICTS OF INTEREST: The authors declare that they have no conflicts of interest.

FINANCIAL SUPPORT: This work has not received any contribution, grant or scholarship.

\section{REFERÊNCIAS}

1. Dunnick NR Report of the 2002 Intersociety Commission meeting: Radiology 2002-today's research is tomorrow's practice. AJR Am J Roentgenol. 2003;180:925-8.

2. Morgan TA, Avrin DE, Carr CD, Dreyer KJ, Flanders AE, Khorasani R, et al. Meaningful use for radiology: current status and future directions. Radiology. 2013;269:318-21. doi: 10.1148/ radiol.13131034.

3. Gillies RJ, Kinahan PE, Hricak H. Radiomics: images are more than pictures, they are data. Radiology. 2016;278:563-77. doi: 10.1148/radiol.2015151169.

4. Sala E, Mema E, Himoto Y, Veeraraghavan H, Brenton JD, Snyder A, et al. Unravelling tumour heterogeneity using next-generation imaging: radiomics, radiogenomics, and habitat imaging. Clin Radiol. 2017;72:3-10. doi: 10.1016/j. crad.2016.09.013.

5. Bello J. Turf issues in radiology and its subspecialties. Neuroimaging Clin N Am. 2012;22:411-9. doi: 10.1016/j. nic.2012.04.006.

6. European Society of Radiology (ESR). Medical imaging in personalised medicine: a white paper of the research committee of the European Society of Radiology (ESR). Insights Imaging. 2015;6:141-55. doi: 10.1007/s13244-015-0394-0. 